\title{
A Model of Gas Recycling Based on Condensed $\mathbf{H}_{2}$
}

\author{
Daniel Pfenniger \\ University of Geneva, Geneva Observatory, Switzerland
}

\begin{abstract}
To address, among other questions, puzzling observations about star forming in the extreme outer HI disk of M31 (Cuillandre et al. 2001), a scenario of interstellar gas cycling between the visible and a very cold invisible phase is investigated. The key new element sketched here, allowing to maintain the bulk of the gas out of sight, is that molecular hydrogen becomes liquid or solid below $33 \mathrm{~K}$ at sufficiently high pressure, allowing AU-sized spheres of very cold gas to be stabilised by incompressible cores of condensed $\mathrm{H}_{2}$ (Pfenniger 2004). These predicted cold gas globules are relatively weakly bound $\left(\sim 10^{-3} \mathrm{eV} /\right.$ nucleon $)$, such that their lifetime depends directly on the ambient UV/CR excitation level. At galactic scale the globules behave as collisionless bodies, and evaporate and become the usual visible ISM gas through heating. Much of the ISM gas can thus spend a long time in this cold condensed phase in low excitation regions. N-body simulations of galactic disks modeling such effects have been run, and some of their features are described in more detail in this volume and elsewhere (Revaz \& Pfenniger 2004).
\end{abstract}

\section{Introduction}

Despite enormous observational advances over several decades, the major problems of galactology remain the same, i.e.: 1) What are galaxies mostly made of? 2) How do galaxies form and evolve? 3) How does the ISM work? 4) What are the rules of star formation? Such a long stagnation in the basic understanding of galaxies is a strong motivation to re-examine accepted assumptions.

Indeed, the list of physical paradoxes in the ISM is long, despite the fact that they concern classical physics. It is well established that the cold ISM is highly clumped down to at least milli-pc scales, has short cooling times, and its supersonic turbulence is ubiquitous, even in cold clouds. The immediate consequence of supersonic turbulence is that the ISM gas must be generally out of pressure equilibrium, that is, also out of dynamical and thermal equilibrium: the traditional assumptions of thermal physics are not even granted. Deep in the non-linearity following gravitational instability is the star formation process which depends on the poorly understood but observed mass fragmentation, and subsequent collapse. In many cases sub-stellar mass fragmentation is observed, such as the not understood cometary globules in planetary nebulae, or the filamentary structures in supernovae remnants.

Old theoretical expectations have not been satisfactory explained. The very short ISM gas cooling time (kyr) in inhomogeneous conditions leads to 
the bold expectation that fragmentation and gravitational collapse should occur very often and very fast. This outcome is dismissed on observational grounds: since the ISM is not fully turned into stars, "obviously" the bold expectation of fast collapse must be wrong. However, further studies continue to indicate that collapse and singularities cannot be easily avoided, e.g., by magnetic fields, and occur on short time-scales (e.g., Klessen et al. 2000; Huber \& Pfenniger 2002). Since no general star formation is observed, commonly accepted assumptions must deserve re-examination.

At galactic scales, recent work has revealed puzzling relationships of $\mathrm{HI}$ with stars, from which one can argue that the visible HI appears to be the photo-dissociated product of an undetected molecular phase (Smith et al. 2000). The mere existence of blue stars in the extreme outer HI disk of M31 is clear evidence that gas fragmentation, and the formation of $\mathrm{H}_{2}$ is effective in situ without the simultaneous detection of CO emission (Cuillandre et al. 2001). The concomitant dust extinction in $\mathrm{HI}$ corroborates the possibility to form $\mathrm{H}_{2}$.

The relationship of HI with dark matter (Bosma 1981; Hoekstra et al. 2001) remains to be explained. The extended HI disk of NGC 2915 (Bureau et al. 1999; Masset \& Bureau 2003) where the stellar component plays a negligible dynamical role offers a particularly good constraint on the relationship of dark matter with HI. In this galaxy the Toomre $Q$ and $X$ parameters, derived assuming all the visible HI mass composes the disk surface density, are both too high by a factor $\sim 5-8$ to match the observed morphology of the HI spiral arms, even assuming the extreme low velocity dispersion of $\mathrm{HI}$ for the dynamical mass. In our view, the simplest way to obtain consistent $Q$ and $X$ in this galaxy is to increase the disk density to several times the HI mass.

At large scales, the ISM dynamics is better represented as a weakly collisional medium, different from the traditional collisional dynamics used in SPH or grid based numerical methods. Some evidences suggesting this are:

a) Barred galaxy rings, which are well reproduced with long mean free-path sticky particles methods (Schwarz 1981), but never reproduced with SPH or grid methods.

b) The detection of gas in Cen A stellar shells (Charmandaris, Combes \& van der Hulst 2000), since stellar shells are supposed to be self-crossing collisionless structures.

c) Gaseous bars in spirals (e.g., the "bar" in NGC 2915 HI disk, Bureau et al. 1999) because in a collisional gas medium (a proto-stellar disk) a bar is short lived and fragments into a binary condensation.

\section{Gravitational Collapse}

Central to the discussion of the fate of cold gas is the outcome of gravitational collapse, because a collapse is often usually associated to formation of hot bodies such as stars. Below we show how the opposite outcome, bodies based on a cold core, is another possibility.

In classical spherical collapse studies (e.g., Larson 1969), one learns that a spherical collapse of an initial cold $(T \approx 10 \mathrm{~K})$ ISM gas cloud increases its central density almost isothermally towards infinity in a finite time, a singular event. Isothermality results from the large transparency of the cold ISM to its own 
thermally generated photons, such that the cooling time remains much shorter than the free-fall time. However in the growing density collapsing core, an optical depth exceeding unity is finally reached, allowing the core to keep thermal photons longer, which leads central temperature and pressure to increase, stopping the singular density growth. All this supposes the gas equation of state remains perfect all the way.

Let us estimate the central density at which the centre of a pseudo isothermal sphere

$$
\rho(r)=\frac{\rho_{0}}{1+\left(r^{2} / r_{c}^{2}\right)}, \quad M(r)=4 \pi \rho_{0} r_{c}^{3}\left[\frac{r}{r_{c}}-\arctan \left(\frac{r}{r_{c}}\right)\right],
$$

becomes opaque for constant opacity $\kappa$. The optical depth from the center to infinity is:

$$
\tau_{\infty}=\int_{0}^{\infty} \kappa \rho(r) d r=\frac{\pi}{2} \kappa \rho_{0} r_{c} .
$$

Requiring that $\tau_{\infty}=1$ and that the pseudo isothermal sphere is in dynamical equilibrium,

$$
\lim _{r \rightarrow \infty} \frac{G M(r)}{r}=\frac{3 k T}{\mu m_{p}}
$$

leads to a simple expression for the core radius and the critical central density above which the centre begins to be opaque:

$$
r_{c}=\frac{3 \kappa k T}{8 G \mu m_{p}}, \quad \rho_{0}=\frac{16 G \mu m_{p}}{3 \pi \kappa^{2} k T} \approx 2.7 \cdot 10^{-8} \frac{(\mu / 2)}{\left(\kappa / 10^{-4}\right)^{2}(T / 10)}\left[\mathrm{g} \mathrm{cm}^{-3}\right] .
$$

This critical density is independent of the mass or size of the cloud. Now, for general equations of state the temperature increase in a homologous collapse is related to the density increase (see, e.g., Padmanabhan 2001 [Fig. 3.7]):

$$
\frac{d T}{T}=\left(\frac{4 \alpha-3}{3 \delta}\right) \frac{d \rho}{\rho}, \quad \text { where } \quad \alpha \equiv\left(\frac{\partial \ln \rho}{\partial \ln P}\right)_{T}, \quad \delta \equiv-\left(\frac{\partial \ln \rho}{\partial \ln T}\right)_{P} .
$$

So when the isothermal compressibility $\alpha$ decreases below $3 / 4$, further compression leads to cooling, a reversal of the usual perfect gas behaviour. In usual stellar or planetary conditions compressibility decreases when matter is subject to electron degeneracy, but the same effect holds for any hardly compressible matter: it cools down when compressed.

Now molecular hydrogen, the bulk of cold gas, becomes liquid or solid below $33 \mathrm{~K}$ at sufficient pressure. The $\mathrm{H}_{2}$ critical point occurs at $T_{c}=33 \mathrm{~K}, P_{c}=$ $13 \mathrm{bar}$, while the triple point at $T_{3}=14 \mathrm{~K}, P_{3}=0.07 \mathrm{bar}$. At low $T$, the saturation pressure and gas density drops to interstellar values. Between 1 and $14 \mathrm{~K}$ the sublimation curve is well approximated by (Pfenniger \& Combes 1994):

$$
P_{\text {solid-gas }} \approx 5.7 \cdot 10^{20} T^{5 / 2} \mathrm{e}^{-91.75 / T}\left[\mathrm{~K} \mathrm{~cm}^{-3}\right] .
$$

An analytical expression for the full $\mathrm{H}_{2}$ equation of state $\rho(P, T)$ in cold condition has been derived in the temperature range $[1-100] \mathrm{K}$, from cosmic pressures up to $1 \mathrm{kbar}$, by gathering laboratory data from the literature (Pfenniger 2004). 


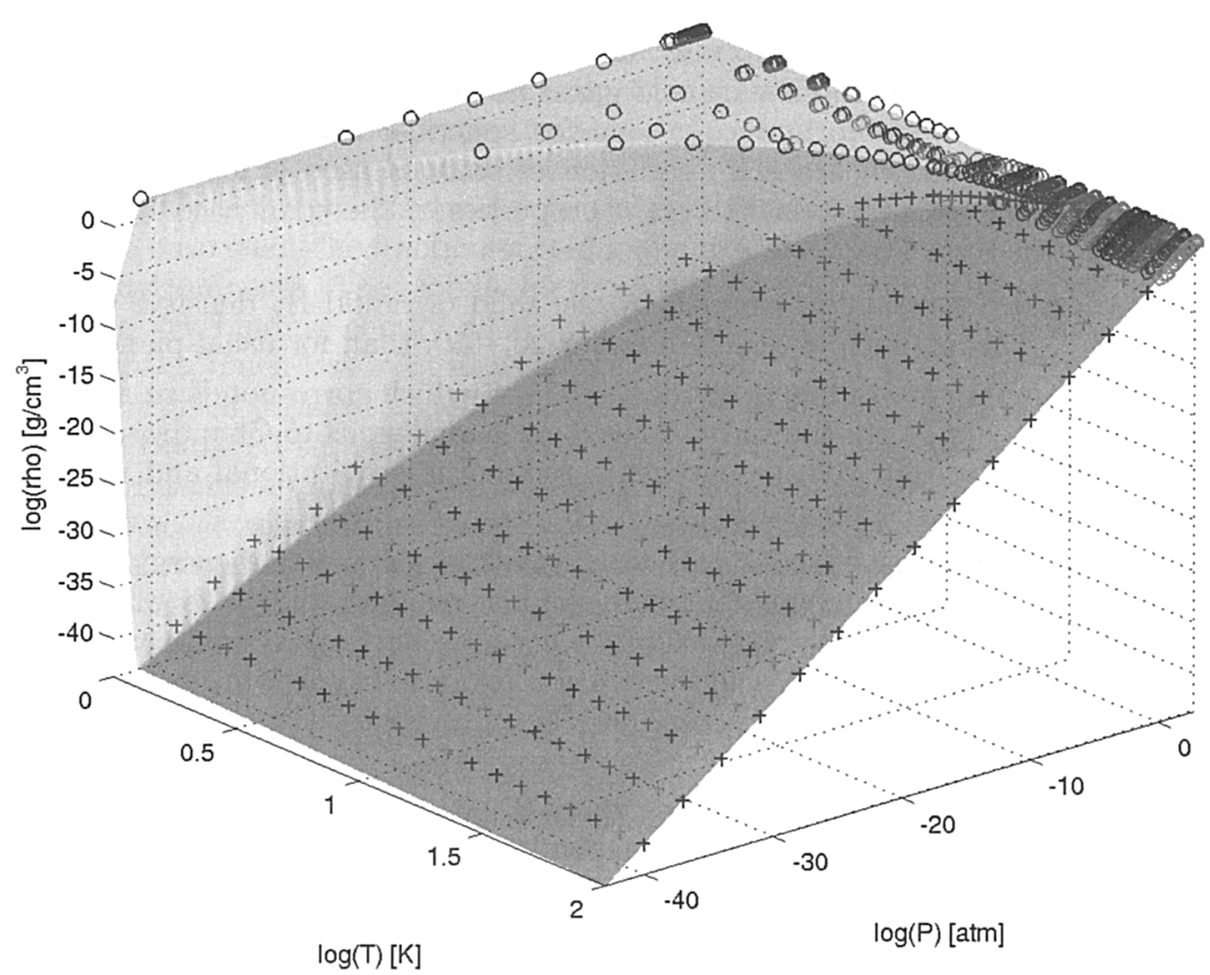

Figure 1. $\mathrm{H}_{2}$ equation of state in the range $1<T<100 \mathrm{~K}$ and $10^{-43}<P<10^{3}$ bar (Pfenniger 2004)

Fig. 1 shows the analytical fit to the indicated data points displaying the steep density wall that occurs below $33 \mathrm{~K}$, breaking the perfect gas inclined plane.

The $\mathrm{H}_{2}$ gas density $\rho$ at the critical pressure is almost the perfect gas density for the partial pressure $P_{\text {solid-gas. Equating this density } \rho \text { to the critically opaque }}$ density $\rho_{0}$ above provides the critical opacity $\kappa_{\text {crit }}$ below which $\mathrm{H}_{2}$ becomes incompressible before becoming opaque to its thermal radiation:

$$
\kappa_{\text {crit }} \approx 1.3 \cdot 10^{-3}(T / 5)^{-5 / 4} \mathrm{e}^{9.18 /(T / 5)}\left[\mathrm{cm}^{2} \mathrm{~g}^{-1}\right] .
$$

Since the ISM submm opacities typical of low metallicity cold clouds can be as low as $\sim 10^{-3}\left[\mathrm{~cm}^{2} / \mathrm{g}\right.$ ] or less (see Draine 2004, [Fig. 12]), at least below $\sim 5 \mathrm{~K}$ a collapsing cloud is able to condense $\mathrm{H}_{2}$ in the core before becoming opaque. Further compression of the condensed core, instead of heating it, cools it. The condensed core thus is able to stop a collapse without becoming hot. Somewhat warmer or more opaque initial gas can still reach $\mathrm{H}_{2}$ condensation despite a temporary temperature increase provided the core remains below $33 \mathrm{~K}$.

We arrive thus at the surprising possibility that gravitational collapse in cold conditions leads not necessarily to a body with a hot centre, such as planets or stars, but to a temperature inverted gas globule with a condensed cold core. The 
core is made of solid $\mathrm{H}_{2}$ below $14 \mathrm{~K}$, or liquid $\mathrm{H}_{2}$ above, up to $33 \mathrm{~K}$. Around the core an $\mathrm{H}_{2}$-He gaseous atmosphere may extend to distances much larger than the core size, and thus makes up most of the globule mass.

The typical properties of such cold gas condensations have been calculated by integrating the equilibrium equations for spherical isothermal gravitating bodies for which the accurate $\mathrm{H}_{2}$ equation of state is used (Pfenniger 2004). Summarizing the calculations, the main properties of the condensed $\mathrm{H}_{2}$ based globule models are:

a) The central density is determined by the solid or liquid $\mathrm{H}_{2}$ density: $0.07-$ $0.09 \mathrm{~g} \mathrm{~cm}^{-3}$. This is much lower, by a factor $20-50$, than for usual planets.

b) The maximum core radius is about $2 \cdot 10^{4} \mathrm{~km}$, which corresponds to a maximum core mass similar to the Earth mass (this is analogous to Chandrasekhar's mass for white dwarfs). At this maximum radius the gravitational and van der Waals binding energies are similar.

c) The cold globules have a relatively weak gravitational binding energy: about $0.001 \mathrm{eV} /$ nucleon, which means that such globules can be rapidly evaporated by sufficient radiative or cosmic ray excitation, but the densest parts, especially the cores, can survive several Gyr in low excitation regions. In other terms, repeated cycles of collapse/evaporation trap an increasingly large fraction of the gas mass in condensed cores.

d) An extended atmosphere, extending up to $3-30 \mathrm{AU}$ for a Jupiter mass, has rapidly an isothermal profile $\propto r^{-2}$, independently of the central core pressure. The atmosphere is expected to be transparent to submm-FIR radiation, but opaque to visible/UV radiation (Combes \& Pfenniger 1997).

It should be noted that the external "heating" of cold globules by penetrating radiation (FIR, cosmic rays) does not increase their apparent temperature due to the negative specific capacity of self-gravitating objects. Such "heating" expands and evaporates the atmosphere without increasing temperature, i.e., without particular emission signature.

\section{Including Cold Globules in a Simple Galaxy Model}

The previous results allow us to set up a simplified galaxy model in which we wish to study the interactions and mass exchanges of three baryonic mass phases:

a) A dark gas phase made of collisionless cold $\mathrm{H}_{2}$ globules.

b) A visible gas phase made of traditional, semi-collisional and dissipative gas (HII, HI, molecular clouds). This phase is able to cool, to form stars, and also to condense into the dark gas phase.

c) A collisionless stellar component heating the gas phases by its continuous radiation, and also by the enhanced energy output from massive stars.

A brief description of the numerical simulations is given elsewhere in this volume, and in Revaz \& Pfenniger (2004). It suffices to note here that the simulations deplete rapidly the dark mass component within the optical disk, but keep it for a long time as a gas reservoir in the outer massive cold gas disk. Such a model is able to account for much of the morphological features observed in NGC 2915, such as gaseous spiral arms, and in the outer HI disk of M 31. 


\section{Conclusions}

A general mechanism has been described in which the solid/liquid phases of $\mathrm{H}_{2}$ drive a collapse phase not necessarily toward stars, but toward cold globules with a range of sizes and properties above planetary sizes. Thus collapse can be frequent in the ISM without necessarily leading to the formation of many stars. The initial gas temperature and opacity are the essential factors determining the collapse outcome. The fraction of cold globules should increase in cold and metal poor environments. The globule lifetimes depend on the ambient excitation, the relative low binding energy allows the globule cores to survive several Gyr in outer disks, much less in optical disks.

The given physical ingredients can explain how a cold gas reservoir producing the visible HI subject to UV excitation can exist. The cause of the HI/dark matter relationship and maximum disk decompositions is then explicit, as well as the anti-correlation in the spiral sequence of dark matter and stars. In this scenario, massive dark disk galaxies evolve smoothly from dark gas disks made of collisionless cold globules, and then along the dark matter decreasing spiral sequence from $\mathrm{Sd}$ to $\mathrm{Sa}$, keeping a minor visible gas fraction all the way. By behaving essentially as collisionless bodies, the cold globules' velocity dispersion increases slowly by dynamical heating to values large enough to avoid a massive disk stability problem.

Acknowledgments. This work was supported by the Swiss National Science Foundation.

\section{References}

Bosma, A. 1981, AJ, 86, 1791

Bureau, M., et al. 1999, AJ, 118, 2158

Charmandaris, V., Combes, F., \& van der Hulst, J.M. 2000, A\&A, 356, 1

Combes, F., \& Pfenniger, D. 1997, A\&A, 327, 453

Cuillandre, J.-Ch., et al. 2001, ApJ, 554, 190

Draine, B. T. 2004, in "The Cold Universe", Saas Fee Advanced Course 32, D.

Pfenniger \& Y. Revaz (eds.), Springer-Verlag, Berlin, p. 213

Hoekstra, H., van Albada, T. S., \& Sancisi, R. 2001, MNRAS, 323, 453

Huber, D., \& Pfenniger, D. 2002 A\&A, 386, 359

Klessen, R.S., Heitsch, F., \& Mac Low, M.-M. 2000, ApJ, 535, 887

Larson, R.B. 1969, MNRAS, 145, 271

Masset, F. S., \& Bureau, M. 2003, ApJ, 586, 152

Padmanabhan, T. 2001, Theoretical Astrophysics Vol. II, Cambridge

Pfenniger, D. 2004, A\&A, to be submitted

Pfenniger D., \& Combes F. 1994, A\&A, 285, 93 (PC94)

Revaz, Y., \& Pfenniger, D. 2004, A\&A, to be submitted

Schwarz, M. P. 1981, ApJ, 247, 77

Smith, D.A., et al. 2000, ApJ, 538, 608 